\title{
On the asymptotic behaviour of some Markov processes
}

by

MEHDI MOULINE (Rabat) and BÜI-TRONG-LIỄ U (Lille)

The purpose of this paper is essentially to study the relations between the ergodicity of a Markor process and the ergodicity of Markov processes constructed from the former with the help of a measurable mapping (Part II). The conditions under which this construction is possible have been given in [5]. They are slightly modified here; this is the main object of the first part, to which we add some complements. The terminologyused in this paper is that of [1] and [5].

In the following, we shall denote by $\boldsymbol{N}$ the set of non-negative integers, $\boldsymbol{N}^{*}$ the set of positive integers, and $1_{B}$ the indicator of the set $B$.

\section{MARKOV PROCESSES CONSTRUCTED FROM ANOTHER WITH THE HELP OF A MEASURABLE MAPPING}

I. 0. A $\sigma$-algebra $\mathscr{T}$ of subsets of a non-empty set $\mathscr{Y}$ is said to be separable if there exists a countable family of subsets of $\mathscr{Y}$ generating $\mathscr{T}$. For example, if $\mathscr{Y}$ is a Polish space, and if $\mathscr{T}$ is the $\sigma$-algebra generated by open sets of $\mathscr{Y}$, then $\mathscr{T}$ is separable.

A class $\mathscr{C}$ of subsets of $\mathscr{Y}$ is said to be semi-compact if for every sequence $\left(C_{n}\right)_{n \in \mathbf{N}^{*}}$ of elements of $\mathscr{C}$, such that $\bigcap_{n \in \mathbf{N}^{*}} C_{n}=\varnothing$, there exists an $m \in \boldsymbol{N}^{*}$ such that $\bigcap_{n \leqslant m} C_{n}=\emptyset$.

A class $\mathscr{F}$ of subsets of $\mathscr{Y}$ is said to have the property of approximation relatively to a probability $Q$ and to a $\sigma$-algebra $\mathscr{T}$ if

$$
\forall C \epsilon \mathscr{T}, \quad Q(C)=\sup _{\substack{F \epsilon \mathscr{F} \\ F \subset C}} Q\left(F^{\prime}\right) .
$$

I. I. Let $\left(\mathscr{X}^{\prime}, \mathscr{B}^{\prime}\right)$ and $\left(\mathscr{X}^{\prime \prime}, \mathscr{B}^{\prime \prime}\right)$ be two measurable spaces. We recall that a transition probability $P$ from $\left(\mathscr{X}^{\prime}, \mathscr{B}^{\prime}\right)$ to $\left(\mathscr{X}^{\prime \prime}, \mathscr{B}^{\prime \prime}\right)$ is a mapping $P: \mathscr{X}^{\prime} \times \mathscr{B}^{\prime \prime} \rightarrow[0,1]$ such that $\nabla x \in \mathscr{X}^{\prime}, P(x, \cdot)$ is a probability measure on $\mathscr{B}^{\prime \prime}$, and $\nabla B \in \mathscr{B}^{\prime \prime}, P(\cdot, B)$ is a real random variable defined on $\left(\mathscr{X}^{\prime}, \mathscr{B}^{\prime}\right)$. Let $\left(\left(\mathscr{X}_{t}, \mathscr{B}_{t}\right)\right)_{t \in \mathbf{N}^{*}}$ be a sequence of measurable spaces, $\left(P_{t, t_{+1}}\right)_{t \in \mathbf{N}^{*}}$ a sequence of transition probabilities such that $\nabla t \in N^{*}, P_{t, t+1}$ is a transition 
probability from $\left(\mathscr{X}_{t}, \mathscr{B}_{t}\right)$ to $\left(\mathscr{X}_{t+1}, \mathscr{B}_{t+1}\right)$. We shall denote by $P_{s, t}$ the transition probability from $\left(\mathscr{X}_{s}, \mathscr{B}_{s}\right)$ to $\left(\mathscr{X}_{t}, \mathscr{B}_{t}\right)$ defined by

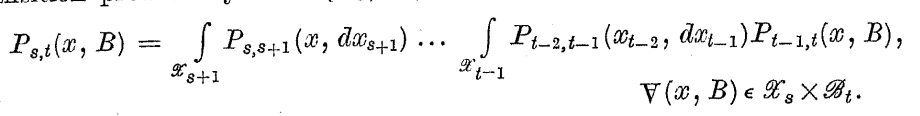

The family $\left(\left(\mathscr{X}_{t}, \mathscr{B}_{t}\right), P_{t, t+1}\right)_{t \in \mathbf{N}^{*}}$ is said to be a Markov process. In the special case $\mathscr{X}_{t}=\mathscr{X}, \mathscr{B}_{t}=\mathscr{B}$ and $P_{t, t+1}=P, \forall t \in \boldsymbol{N}^{*}$, we say that the Markov process is homogeneous, and we denote it by $\left((\mathscr{X}, \mathscr{B}), P_{n}\right)_{n \in \mathbb{N}^{*}}$, where $P_{n}=P_{s, s+n}, \forall s \in \boldsymbol{N}^{*}$.

Let $(\Omega, \mathscr{A}, \operatorname{Pr})$ be a probability space. A Markov random function $\left(X_{t}\right)_{t \in \mathbf{N}^{*}}$ defined on $(\Omega, \mathscr{A}, \operatorname{Pr})$ and taking its values in a measurable space $(\mathscr{X}, \mathscr{B})$ is said to be attached to the Markov process $\left((\mathscr{X}, \mathscr{B}), P_{n}\right)_{n \in \mathbf{N}^{*}}$ if it has $P$ as transition probability.

Let $(\mathscr{Y}, \mathscr{T})$ be a measurable space and $f$ be a $\mathscr{T}$ - $\mathscr{B}$-measurable mapping from $\mathscr{X}$ onto $\mathscr{Y}$. Let us denote by $\mathscr{B}_{f}$, the sub- $\sigma$-algebra $f^{-1}(\mathscr{T})$ of $\mathscr{B}$. As mentionned in the introduction, the purpose of this part is to recall and to modify, slightly, results given by Rosenblatt in [5]. We also give some complements, which will be useful for the second part.

For convenience, we shall define the following hypotheses:

I. 2. (i) $\forall x \in \mathscr{X},\{x\} \in \mathscr{B}$,

(ii) $\nabla y \in \mathscr{Y},\{y\} \in \mathscr{T}$,

(iii) $\forall x \in \mathscr{X}, P_{\mathscr{B}_{f}}(x, \cdot)$ is dominated (Fiz. absolutely continue with respect to) by a positive $\sigma$-finite measure $\mu$ on $\mathscr{B}_{f}, P_{\mathscr{B}_{j}}(x, \cdot)$ being the restriction of $P(x, \cdot)$ to $\mathscr{B}_{f}$.

I. 3. $\mathscr{T}$ is separable and there exists a semi-compact sub-class $\mathscr{F}$ of $\mathscr{T}$ such that, for any probability $Q$ on $\mathscr{T}, \mathscr{F}$ has the property of approximation relatively to $Q$ and to $\mathscr{T}$.

Proofs of propositions I. 10 and I. 11 below will be omitted: they are the same as those given in [5], modulo the following result:

I. 4. Proposition. Let $A \in \mathscr{B}_{f}$, let $\xi$ be a probability on $\mathscr{B}$ and $\xi_{\mathscr{B}_{f}}$ be the restriction of $\xi$ on $\mathscr{B}_{f}$. Under hypothesis I. 3 , there exists a mapping v: $A \times \mathscr{T} \rightarrow[0,1]$ such that

(i) $\nabla C \epsilon \mathscr{T}, \nu(\cdot, C)$ is $\mathscr{B}_{f}^{(A)}$-measurable and $\nabla B \in \mathscr{B}_{f}^{(A)}$,

$$
\int_{B} v(x, C) d \xi(x)=\int_{B} P\left(x, f^{-1}(C)\right) d \xi(x)
$$

$\mathscr{B}_{f}^{(A)}$ denoting the o-algebra $\mathscr{B}_{f} \cap A$

(ii) $\forall x \in A, \nu(x, \cdot)$ is a probability on $\mathscr{T}$.

We omit the proof. In the particular case $A=\mathscr{X}$, we have the

I. 5. CoROLLARY. Under hypothesis I. 3, there exists a mapping $\nu: \mathscr{X} \times \mathscr{T} \rightarrow[0,1]$ such that (i) $\nabla C \epsilon \mathscr{T}, \nu(\cdot, C)$ is a version of the conditional expectation $E_{\xi}\left[P\left(\cdot, f^{-1}(C)\right) \mid \mathscr{B}_{f}\right]$ relatively to $\xi$.

(ii) $\nabla x \in \mathscr{X}, v(x, \cdot)$ is a probability on $\mathscr{T}$.

I. 6. We know (cf. [3]) that: For every family of probabilities dominated by a probability, one can find an equivalent countable subfamily of probabilities. Since the family $\left(\left.P_{\mathscr{B}_{f}}(x, \cdot)\right|_{x_{\epsilon} \mathscr{T}}\right.$ of probabilities is dominated, it is equivalent to a countable sub-family $\left(P_{\mathscr{B}_{f}}\left(x_{i}, \cdot\right)\right)_{i_{\epsilon} \mathbf{N}^{*}}$. The dominating (in fact, equivalent) measure can be taken, and shall be taken, as the probability measure

$$
\mu=\sum_{i \in \mathbf{N}^{*}} a_{i} P_{\mathscr{B} f}\left(x_{i}, \cdot\right), \quad \text { where } \alpha_{i}>0 \text { and } \sum_{i \in \mathbf{N}^{*}} a_{i}=1 .
$$

We shall also make use, in Part II, of the probability measure

$$
\bar{\mu}=\sum_{i \in \mathbf{N}^{*}} a_{i} P\left(x_{i}, \cdot\right) \quad \text { on } \mathscr{B} \text {. }
$$

I. 7. A set $S \in \mathscr{B}_{f}$ is said to be a single entry set if $\mu(S)>0$ and if there exists a point $y \in \mathscr{Y}$ such that $P(x, S)=0, \forall x \in \mathfrak{G} f^{-1}(y)$. Such a point $y$ is called a single entry point corresponding to $S$. A set $S \in \mathscr{B}_{i}$ is said to be a maximal single entry set if it is a single entry set and if there does not exist any single entry set $S$ such that $S^{\prime} \supset S$ and $\mu\left(S^{\prime}\right)>\mu\left(S^{\prime}\right)$.

I. 8. Let us now recall some results:

$1^{0}$ If $S$ is a single entry set, there exists at least one point $x \in \mathscr{X}$ such that $P(x, S)>0$.

$2^{\circ}$ A maximal single entry set $S$ is defined modulo a $\mu$-null set.

$3^{\circ}$ There are at most a countable class of disjoint maximal single entry sets $\left(S_{k}\right)_{k_{\in} \mathbf{N}^{*}}$ and corresponding entry points.

$4^{0}$ Define $M=\mathfrak{C}\left(\bigcup_{k \in N^{*}} S_{l_{c}}\right)$. If $\mu(M)=0$, then $\mathscr{X}$ is the union of an at most countable family of maximal single entry sets.

$5^{\circ}$ If $\mu(M)=0$ and if $S$ is a maximal single entry set such that $P(x, S)>0$, then $P(x, S)=1$.

$6^{\circ}$ If $\mu(M)=0$, and if $y \epsilon \mathscr{Y}$ is such that $\mu\left(f^{-1}(y)\right)>0$, then $f^{-1}(y)$ is a single entry set.

$7^{\circ}$ If $\mu(M)=0$, then $\mathscr{Y}$ is the at most countable set of single entry points.

I. 9. It is to be remarked that if $\left(X_{t}\right)_{t \in \mathbf{N}^{*}}$ is a Markov random function attached to a homogeneous Markov process $\left((\mathscr{X}, \mathscr{B}), P_{n}\right)_{n \in \mathbf{N}^{*}}$, the random function $\left(f \circ X_{t}\right)_{t_{\in} \boldsymbol{N}^{*}}$ is not generally Markovian. Rosenblatt in [5] has given the conditions under which $\left(f \circ X_{t}\right)_{t \in \mathbf{N}^{*}}$ is also Markovian.

I. 10. Proposition. Under hypothesis I. 2, and if $\mu(M)=0$, then whatever be the Markov random function $\left(X_{t}\right)_{t \in \mathbf{N}^{*}}$ attached to the Markov process $\left((\mathscr{X}, \mathscr{B}), P_{n}\right)_{n \in N^{*}}$, the random function $\left(f \circ X_{t}\right)_{t_{\epsilon} N^{*}}$ is Markovian. Studia Mathematica $\mathrm{XXXIV.3}$ 
I. 11. Consider now the case $0<\mu(M)<1$. There exist. some single entry sets, for this existence is equivalent to the condition $\mu(M)<1$. Let $S$ be a single entry set such that we may be able to enter $S$ starting from $M$, that is to say there exist a positive integer $n$ and $n$ points $y_{1}, \ldots$ $\ldots, y_{n} \epsilon \mathscr{Y}$ such that: a) $f^{-1}\left(y_{j}\right), j=2, \ldots, n$, are single entry sets and $f^{-1}\left(y_{1}\right)$ is not; b) $y_{j-1}$ is the single entry point corresponding to $f^{-1}\left(y_{j}\right), j=2, \ldots, n$ and $y_{n}$ is the single entry point corresponding to $S$. The integer $n$ and the points $y_{1}, \ldots, y$, when they exist, are uniquely defined and constitute a thread of length $n$ entering $S$.

I. 12. Proposition. Under the hypotheses I. 2 and I. 3 , a necessary and sufficient condition for $\left(f \circ X_{t}\right)_{t \mathbf{N}^{*}}$ to be Markovian whatever be the Markov random function $\left(X_{t}\right)_{t \in \boldsymbol{N}^{*}}$, attached to the Markov process $((\mathscr{X}, \mathscr{B})$, $\left.P_{n}\right)_{n \in \mathbf{N}^{*}}$, is that:

(i) There exists a mapping $R: M \times \mathscr{T} \rightarrow[0,1]$ such that $\forall C \epsilon \mathscr{T}$, $\forall x_{0} \in \mathscr{X}, R(\cdot, O)$ is $\mathscr{B}_{f}^{(M)}$-measurable and $\nabla B \in \mathscr{B}_{f}^{(M)}$,

$$
\int_{B} P\left(x_{0}, d x_{1}\right) R\left(x_{1}, C\right)=\int_{B} P\left(x_{0}, d x_{1}\right) P\left(x_{1}, f^{-1}(C)\right) .
$$

(ii) If $S$ is a single entry set and if there exists a thread of length $n$ entering $S$, then there exists a mapping $R_{n}: S \times \mathscr{T} \rightarrow[0,1]$ such that $\forall C \epsilon \mathscr{T}, \forall x_{0} \in \mathscr{X}, R_{n}(\cdot, C)$ is $\mathscr{B}_{f}^{(S)}$-measurable and $\nabla B \epsilon \mathscr{B}_{f}^{(S)}$,

$$
\int_{B} P_{n+1}\left(x_{0}, d x_{1}\right) R_{n}\left(x_{1}, C\right)=\int_{B} P_{n+1}\left(x_{0}, d x_{1}\right) P\left(x_{1}, f^{-1}(C)\right) .
$$

As mentioned above, we omit the proof of I.12 and refer to [5]. We simply recall that the absolute probabilities $\tilde{Q}_{t}$ and the transition probabilities $Q_{t, t+1}(\cdot, \cdot), t \in \boldsymbol{N}^{*}$, of the Markov random function $\left(f \circ X_{t}\right)_{t \in \boldsymbol{N}^{*}}$ are constructed as follows: $\nabla C \epsilon \mathscr{T}$.

$1^{0} \pi_{t}$ being the law of $X_{t}, t \in N^{*}, \tilde{Q}_{t}$ is defined by $\tilde{Q}_{t}(C)=\pi_{t}\left[f^{-1}(C)\right]$,

$2^{\circ}$ With regard to the transition probabilities:

If $t=1$, we define, $\forall y \in \mathscr{Y}$ and $\forall C \epsilon \mathscr{T}, Q_{1,2}(y, C)=v_{1}(\cdot, C)$, where $x \in f^{-1}(y)$ and where $\nu_{1}(\cdot, C)$ is a version of the conditional expectation $E_{\pi_{1}}\left[P\left(\cdot, f^{-1}(C)\right) \mid \mathscr{B}_{f}\right]$ which is such that $\forall x \in \mathscr{X}, \nu_{1}(x, \cdot)$ is a probability on $\mathscr{T}$ (such a version exists following I. 5).

If $t \geqslant 2$, we distinguish two cases: define

a) If $y \in f(M)$, then $f^{-1}(y) \subset M$ (for $f^{-1}(y)$ is an atom of $\left.\mathscr{B}_{f}\right)$ and we

$$
Q_{t, t+1}(y, C)=R(x, C), \quad \forall C \epsilon \mathscr{T}, \quad \text { where } x \in f^{-1}(y) .
$$

b) If $y \notin f(M)$, then $f^{-1}(y)$ is included in a maximal single entry set $S$. If there exists a thread of length $n$ entering $S$, we put, $\forall C \in \mathscr{T}$,

$$
Q_{t, t+1}=\left\{\begin{array}{lll}
R_{n}(x, C) & \text { if } & t>n, \text { where } x \in f^{-1}(y), \\
v_{t}(x, C) & \text { if } & t \leqslant n, \text { where } x \in f^{-1}(y),
\end{array}\right.
$$

$v_{t}(\cdot, C)$ denoting a $\mathscr{B}_{f}^{(S)}$-measurable function such that $\nabla B \in \mathscr{B}_{f}^{(S)}$,

$$
\int_{B} d \pi_{t}(x) v_{t}(x, C)=\int_{B} d \pi_{t}(x) P\left(x, f^{-1}(C)\right)
$$

and $\forall x \in \mathscr{X}, \nu(\cdot, C)$ is a probability on $\mathscr{T}$ (such a function exists by I. 3 ).

I. 13. Let us now make a few remarks:

$1^{0} \nabla t \geqslant 2, \forall B \epsilon \mathscr{B}_{f}^{(M)}$ and $\nabla C \epsilon \mathscr{T}$,

$$
\int_{B} d \pi_{t}\left(x_{1}\right) P\left(x_{1}, f^{-1}(C)\right)=\int_{B} d \pi_{t}\left(x_{1}\right) R\left(x_{1}, C\right) .
$$

Indeed,

$$
\begin{aligned}
\int_{B} d \pi_{t}\left(x_{1}\right) P\left(x_{1}, f^{-1}(C)\right) & =\int_{B}\left[\int_{\mathscr{X}} d \pi_{t-1}(x) P\left(x, d x_{1}\right)\right] P\left(x_{1}, f^{-1}(C)\right) \\
& =\int_{\mathscr{X}} d \pi_{t-1}(x)\left[\int_{B} P\left(x, d x_{1}\right) P\left(x_{1}, f^{-1}(C)\right)\right] \\
& =\int_{\mathscr{X}} d \pi_{t-1}(x)\left[\int_{B} P\left(x, d x_{1}\right) R\left(x_{1}, C\right)\right] \\
& =\int_{B}\left[\int_{\mathscr{X}} d \pi_{t-1}(x) P\left(x, d x_{1}\right)\right] R\left(x_{1}, C\right) \\
& =\int_{B} d \pi_{t}\left(x_{1}\right) R\left(x_{1}, C\right) .
\end{aligned}
$$

If $f^{-1}(y) \in \mathscr{B}_{f}^{(M)}$ and if $\pi_{t}\left[f^{-1}(y)\right]>0$, then

(I.13.2) $\quad R(x, C)=\frac{1}{\pi_{t}\left[f^{-1}(y)\right]} \int_{f^{-1}(y)} d \pi_{t}\left(x_{1}\right) P\left(x_{1}, f^{-1}(C)\right), \quad \nabla x \in f^{-1}(y)$.

Indeed, $R(\cdot, C)$ is constant on $f^{-1}(y)$ which is an atom of $\mathscr{B}_{f}^{(M)}$. We then make use of (I.13.1) by taking $f^{-1}(y)$ in place of $B$.

$2^{\circ}$ Similarly, we have: $\forall t \geqslant n+2, \nabla B \epsilon \mathscr{B}_{f}^{(S)}$ and $\nabla C \epsilon \mathscr{T}$,

$$
\int_{B} d \pi_{t}\left(x_{1}\right) P\left(x_{1}, f^{-1}(C)\right)=\int_{B} d \pi_{t}\left(x_{1}\right) R_{n}\left(x_{1}, C\right) .
$$

If $f^{-1}(y) \in \mathscr{B}_{f}^{(S)}$ and if $\pi_{t}\left[f^{-1}(y)\right]>0$, then

(I.13.4) $\quad R_{n}(x, C)=\frac{1}{\pi_{t}\left[f^{-1}(y)\right]} \int_{f^{-1}(y)} d \pi_{t}\left(x_{1}\right) P\left(x_{1}, f^{-1}(C)\right), \quad \forall x \in f^{-1}(y)$.

$3^{\circ}$ If $\forall x_{0} \epsilon \mathscr{X}, \forall B \in \mathscr{B}_{j}$ and $\nabla C \epsilon \mathscr{T}$,

(I.13.5) $\quad \int_{B} P\left(x_{0}, d x_{1}\right) P\left(x_{1}, f^{-1}(C)\right)=\int_{B} P\left(x_{0}, d x_{1}\right) R\left(x_{1}, C\right)$, 
where $R(\cdot, C)$ is $\mathscr{B}_{f}$-measurable. Then, we have $\nabla x_{0} \epsilon \mathscr{X}, \nabla B \epsilon \mathscr{B}_{f}, \nabla C \epsilon \mathscr{T}$ and $\nabla n \in \mathcal{N}^{*}$,

(I.13.6)

$$
\begin{aligned}
\int_{B} P_{n+1}\left(x_{0}, d x_{1}\right) & P\left(x_{1}, f^{-1}(C)\right) \\
& =\int_{B}\left[\int_{\mathscr{X}} P_{n}\left(x_{0}, d x\right) P\left(x, d x_{1}\right)\right] P\left(x_{1}, f^{-1}(O)\right) \\
& =\int_{\mathscr{X}} P_{n}\left(x_{0}, d x\right)\left[\int_{\mathcal{B}} P\left(x, d x_{1}\right) P\left(x_{1}, f^{-1}(C)\right)\right] \\
& =\int_{\mathscr{X}} P_{n}\left(x_{0}, d x\right)\left[\int_{B} P\left(x, d x_{1}\right) R\left(x_{1}, C\right)\right] \\
& =\int_{B} P_{n+1}\left(x_{0}, d x_{1}\right) R\left(x_{1}, C\right) .
\end{aligned}
$$

So, (I.13.5) implies I.12 (ii): it is sufficient to take $R_{n}=R$.

$4^{\circ}$ A sufficient condition for I.12 (i) and I.12 (ii) to be satisfied is that $P\left(\cdot, f^{-1}(C)\right)$ is $\mathscr{B}_{f}$-measurable, $\forall C \in \mathscr{T}$.

Indeed, (I.13.5) is then verified, with $P\left(\cdot, f^{-1}(C)\right)$ in place of $R(\cdot, C)$.

I. 14. Consider now the homogeneous case. $\left(X_{t}\right)_{t_{\epsilon} \boldsymbol{N}^{*}}$ and $\left(f \circ X_{t}\right)_{t_{\epsilon} \boldsymbol{N}^{*}}$ having the same meanings as above, it is known that $\left((\mathscr{Y}, \mathscr{T}), Q_{t, t+1}\right)_{t \in \mathbf{N}^{*}}$ is not necessarily homogeneous, although $\left((\mathscr{X}, \mathscr{B}), P_{n}\right)_{n \in \mathbf{N}^{*}}$ is.

A sufficient condition for $\left((\mathscr{Y}, \mathscr{T}), Q_{t, t+1}\right) t_{\in \mathbf{N}^{*}}$ to be homogeneous is that $P(\cdot, B)$ is $\mathscr{B}_{f}$-measurable, $\nabla B \in \mathscr{B}_{f}$.

In the case where there exists no single entry set, this sufficient condition is also necessary.

I. 15. The following remarks will be useful for Part II.

$1^{0}$ Suppose that I.12 (i) and (ii) are verified. Let $v_{s, t}$ be defined by $v_{s, t}(x, C)=Q_{s, t}(y, C)$ for $y \in \mathscr{Y}, x \in f^{-1}(y)$ and $C \in \mathscr{T}$. Then $v_{s, t}(\cdot, C)$ is a version of the conditional expectation $E_{\pi_{s}}\left[P_{t-s}\left(\cdot, f^{-1}(C)\right) \mid \mathscr{B}_{f}\right]$.

Indeed, $\nabla C$ and $C^{\prime} \epsilon \mathscr{T}$, and $\nabla s, t \in \boldsymbol{N}^{*}$ with $s<t$, we have

$$
\operatorname{Pr}\left\{Y_{s} \epsilon C^{\prime}, Y_{t} \in O\right\}=\operatorname{Pr}\left\{X_{s} \epsilon f^{-1}\left(C^{\prime}\right), X_{t} \in f^{-1}(C)\right\},
$$

\section{that is to say}

$$
\int_{C^{\prime}} d \tilde{Q}_{s}(y) Q_{s, t}(y, C)=\int_{f-1\left(C^{\prime}\right)} d \pi_{s}(x) P_{t-s}\left(x, f^{-1}(C)\right) .
$$

Since $v_{s, t}(\cdot, C)$ is $\mathscr{B}_{f}$-measurable, we have

$$
\int_{\sigma^{\prime}} d \tilde{Q}_{s}(y) Q_{s, t}(y, C)=\int_{f-\mathcal{I}_{\left(C^{\prime}\right)}} d \pi_{s, \mathscr{B}_{f}}(x) \nu_{s, t}\left(x, C^{\prime}\right),
$$

$\pi_{s, \mathscr{B}_{f}}$ denoting the restriction of $\pi_{s}$ to $\mathscr{B}_{f}$. Then

$$
\int_{f-1_{\left(O^{\prime}\right)}} d \pi_{s, \mathscr{D}_{f}}(x) v_{s, t}(x, O)=\int_{f-1_{\left(O^{\prime}\right)}} d \pi_{s}(x) P_{t-s}\left(x, f^{-1}(C)\right) .
$$

$2^{\circ}$ Suppose that (I.13.5) is verified. Let $R^{\prime}\left(x, f^{-1}(C)\right)=R(x, C)$, $C \in \mathscr{T} ;\left(R^{\prime}(x, \cdot)\right.$ is then a positive measure on $\left.\mathscr{B}_{f}\right)$ and

$$
\begin{gathered}
R^{(1)}(x, C)=R(x, C), \\
R^{(n)}(x, C)=\int_{\mathscr{X}} R^{\prime}\left(x, d x^{\prime}\right) R^{(n-1)}\left(x^{\prime}, C\right) .
\end{gathered}
$$

Then $\nabla B \epsilon \mathscr{B}_{f}, \nabla t \epsilon \boldsymbol{N}^{*}$ and $\nabla\left(x_{0}, C\right) \epsilon \mathscr{X} \times \mathscr{T}$,

$$
\int_{B} P\left(x_{0}, d x\right) P_{t}\left(x, f^{-1}(C)\right)=\int_{B} P\left(x_{0}, d x\right) R^{(t)}(x, C) .
$$

We proceed by induction. Suppose that the equality is true for $n-\mathbf{1}$, then $\forall B \in \mathscr{B}_{f}$,

$$
\begin{aligned}
\int_{B} P\left(x_{0}, d x\right) P_{n}\left(x, f^{-1}(C)\right) & =\int_{B} P\left(x_{0}, d x\right)\left[\int_{\mathscr{X}} P\left(x, d x^{\prime}\right) P_{n-1}\left(x^{\prime}, f^{-1}(C)\right)\right] \\
& =\int_{B} P\left(x_{0}, d x\right)\left[\int_{\mathscr{X}} P\left(x, d x^{\prime}\right) R^{(n-1)}\left(x^{\prime}, C\right)\right] \\
& =\int_{\mathscr{X}}\left[\int_{B} P\left(x_{0}, d x\right) P\left(x, d x^{\prime}\right)\right] R^{(n-1)}\left(x^{\prime}, C\right) \\
& =\int_{\mathscr{X}}\left[\int_{B} P\left(x_{0}, d x\right) R^{\prime}\left(x, d x^{\prime}\right)\right] R^{(n-1)}\left(x^{\prime}, C\right) \\
& =\int_{B} P\left(x_{0}, d x\right) R^{(n)}(x, C) .
\end{aligned}
$$

$3^{\circ} \nabla s \geqslant 2, \nabla t>s$ and $\nabla C \epsilon \mathscr{T}$, we have $\mu$-a.s.

(I.15.3)

$$
Q_{s, t}(y, C)=R^{(t-s)}(x, C), \quad x \in f^{-1}(y) .
$$

At first, from I.15.2, we have $\nabla B \epsilon \mathscr{B}_{f}$,

$$
\int_{B} d \bar{\mu}(x) P_{n}\left(x, f^{-1}(C)\right)=\int_{B} d \bar{\mu}(x) R^{(n)}(x, C)=\int_{B} d \mu(x) R^{(n)}(x, C),
$$

for $R^{(n)}(\cdot, C)$ is $\mathscr{B}_{f}$-measurable.

Analogously, as in (I.13.3), we have: $\nabla B \in \mathscr{B}_{f}, \nabla s \geqslant 2, \nabla t>s$ and $\forall C \in \mathscr{T}$

$$
\begin{aligned}
\int_{B} d \pi_{s}(x) P_{t-s}\left(x, f^{-1}(C)\right) & =\int_{B} d \pi_{s}(x) R^{(t-s)}(x, C) \\
& =\int_{B} d \pi_{s, \mathscr{B}_{f}}(x) R^{(t-s)}(x, C) .
\end{aligned}
$$

Hence, by $1.15 .1^{\circ}$,

$$
v_{s, t}(\cdot, C)=R^{(t-s)}(\cdot, C), \quad \pi_{s, \mathscr{B}_{f}}-\mathrm{a} . \mathrm{s.}
$$

and then, $\pi_{s, \mathscr{x}_{f}}$-a.s.

$$
Q_{s, t}(y, C)=R^{(t-s)}(x, C), \quad x \in f^{-1}(y)
$$


On the other hand, $\nabla s \geqslant 2, \pi_{s, \mathscr{B}_{f}}$ is dominated by $\mu$, so that we have, $\mu$-a.s.

$$
Q_{s, t}(y, C)=R^{(t-s)}(x, C), \quad x \in f^{-1}(y)
$$

\section{PROBLemS OF ERGODicity}

II. 1. We recall the following definitions (cf. [1]): A Markov process $\left(\Lambda, \mathscr{L}, q_{t, t+1}\right)_{t \in \mathbb{N}^{*}}$ is said to be:

(i) strongly ergodic if $\nabla s \in \boldsymbol{N}^{*}, \nabla z \in \Lambda$ and $\nabla A \in \mathscr{L}, \lim _{t \rightarrow \infty} q_{s, t}(z, A)$ $=\xi_{s}(A)$, where $\xi_{s}$ is a probability on $\mathscr{L}$;

(ii) weakly ergodio if $\nabla s \in \boldsymbol{N}^{*}, \nabla z$ and $z^{\prime} \in \Lambda$ and $\nabla A \in \mathscr{L}, \lim _{t \rightarrow \infty}\left[q_{s, t}(z, A)-\right.$ $\left.-q_{s, t}\left(z^{\prime}, A\right)\right]=0$;

(iii) strongly (resp. weakly) and uniformly ergodic if it is strongly (resp. weakly) ergodic and if, moreover, the limit in (i) (resp. (ii)) holds uniformly with respect to $z$ and $A$.

II. 2. HYPотнеSIS. We suppose that (I. 13.5) is verified, that is to say: there exists a mapping $R: \mathscr{X} \times \mathscr{T} \rightarrow[0,1]$ such that $\nabla C \epsilon \mathscr{T}, R(\cdot, C)$ is $\mathscr{B}_{f}$-measurable and that

$$
\int_{B} P\left(x_{0}, d x_{1}\right) P\left(x_{1}, f^{-1}(C)\right)=\int_{B} P\left(x_{0}, d x_{1}\right) R\left(x_{1}, C\right),
$$

$\nabla x_{0} \in \mathscr{X}, \nabla C \epsilon \mathscr{T}$ and $\nabla B \epsilon \mathscr{B}_{t}$.

By I.13.3 ${ }^{\circ}$, we see that I.12 (ii) is verified.

II. 3. $\forall O \epsilon \mathscr{T}, R(\cdot, C)$ is a version of $E_{P\left(x_{0}, \cdot\right)}\left[P\left(\cdot, f^{-1}(C)\right) \mid \mathscr{B}_{f}\right]$, then $R(\cdot, C)$ is also a version of $E_{\bar{\mu}}\left[P\left(\cdot, f^{-1}(C)\right) \mid \mathscr{B}_{f}\right]$. We have seen (Part I) that we can choose $R$ in such a way that $\nabla x \in \mathscr{X}, R(x, \cdot)$ is a probability on $\mathscr{T}$, and that for $s \geqslant 2, \mu$-a.s.,

$$
Q_{s, t}(y, C)=R^{(t-s)}(x, O), \quad x \in f^{-1}(y),
$$

where $R^{(t-s)}(\cdot, C)$ is a version of $E_{\bar{\mu}}\left[P_{t_{-s}}\left(\cdot, f^{-1}(C)\right) \mid \mathscr{B}_{f}\right]$.

More precisely, there exists a set $N_{f} \in \mathscr{B}_{f}$ (viz. there exists $N \in \mathscr{T}$ such that $N_{f}=f^{-1}(N)$ ), with $\mu\left(N_{f}\right)=0$, such that $\nabla s \geqslant 2, \nabla t \geqslant s, \nabla C \epsilon \mathscr{T}$,

$$
Q_{s, t}(y, C)=R^{(t-s)}(x, C), \quad x \in f^{-1}(y), \text { if } y \notin N \text {. }
$$

We specially consider the case where, $\nabla s \geqslant 2$,

$$
\begin{array}{ll}
Q_{s, t}(y, C)=R^{(t-s)}(x, C), & x \in f^{-1}(y), \quad \text { if } y \notin N ; \\
Q_{s, s+1}(y, C)=\mu^{\prime}(C) \quad \text { if } \quad y \in N, \mu^{\prime} \text { denoting the image of } \mu \text { by } f .
\end{array}
$$

II. 4. Proposition. Onder Hypothesis II.2, if $\left(X_{t}\right)_{t \mathrm{~N}^{*}}$ is a Markov random function attached to a Markov process $\left((\mathscr{X}, \mathscr{B}), P_{n}\right)_{n \in \boldsymbol{N}^{*}}$ strongly and uniformly ergodic, then $\left(f \circ X_{t}\right)_{t \in \mathbf{S}^{*}}$ is a Markov random function attached to a strongly and uniformly ergodic Markov process.

Proof. $1^{\circ}$ By hypothesis, $\lim P_{t}(x, A)=\xi(A)$ uniformly with respect to $x$ and $A$, where $\xi$ is a probability on $\mathscr{B}$. Let $\mathscr{T}_{0}=\left(O_{0}^{i}\right)_{i \in N^{*}}$ be an algebra generating $\mathscr{T} \cdot \nabla C_{0}^{i} \in \mathscr{T}_{0}, R^{(t)}\left(\cdot, C_{0}^{i}\right)$ is a version of the conditional expectation $E_{\bar{\mu}}\left[P_{t}\left(\cdot, f^{-1}\left(C_{0}^{i}\right)\right) \mid \mathscr{O}_{f}\right]$. Since $0 \leqslant P_{t}\left(\cdot, f^{-1}\left(C_{0}^{i}\right)\right) \leqslant 1$, $\nabla t \in \boldsymbol{N}^{*}$, we have

$$
\lim _{t \rightarrow \infty} R^{(t)}\left(\cdot, O_{0}^{i}\right)=E_{\bar{\mu}}\left[\lim _{t \rightarrow \infty} P_{t}\left(\cdot, f^{-1}\left(O_{0}^{i}\right)\right) \mid \mathscr{B}_{f}\right]=\xi\left[f^{-1}\left(C_{0}^{i}\right)\right], \quad \mu_{\text {-a.s. }}
$$

But $\mathscr{T}_{0}$ is countable, so that there exists a set $N_{1} \in \mathscr{O}_{f}$ such that $\mu\left(N_{1}\right)=0$ and

(II.4.1) $\quad \lim _{t \rightarrow \infty} R^{(t)}\left(x, C_{0}^{i}\right)=\xi\left[f^{-1}\left(C_{0}^{i}\right)\right], \quad \nabla x \notin N_{1}$ and $\nabla C_{0}^{i} \epsilon \mathscr{T}_{0}$.

Let us now prove that this limit is uniform, $\mu$-a.s. Indeed, by hypothesis, $\nabla \varepsilon>0$, 且 $n_{\varepsilon} \in \boldsymbol{N}^{*}$ such that $t>n_{\varepsilon}$ implies $-\varepsilon+\xi\left[f^{-1}\left(C_{0}^{i}\right)\right]<P_{t}\left(x, f^{-1}\left(C_{0}^{i}\right)\right)<\varepsilon+\xi\left[f^{-1}\left(C_{0}^{i}\right)\right], \quad \nabla\left(x, C_{0}^{i}\right) \epsilon \mathscr{X} \times \mathscr{T}_{0}$.

Hence, $\nabla B \in \mathscr{B}_{f}$,

$$
\left(-\varepsilon+\xi\left[f^{-1}\left(C_{0}^{i}\right)\right]\right) \cdot \mu(B)<\int_{B} d \mu(x) P_{t}\left(x, f^{-1}\left(C_{0}^{i}\right)\right)<\left(\varepsilon+\xi\left[f^{-1}\left(C_{0}^{i}\right)\right]\right) \cdot \mu(B),
$$

and, consequently, $\nabla B \epsilon \mathscr{B}_{f}$,

$$
\left(-\varepsilon+\xi\left[f^{-1}\left(C_{0}^{i}\right)\right]\right) \cdot \mu(B)<\int_{B} d \mu(x) R^{(t)}\left(x, C_{0}^{i}\right)<\left(\varepsilon+\xi\left[f^{-1}\left(C_{0}^{i}\right)\right]\right) \cdot \mu(B) .
$$

This implies that

(II.4.2) $-\varepsilon+\xi\left[f^{-1}\left(C_{0}^{i}\right)\right]<R^{(t)}\left(\cdot, C_{0}^{i}\right)<\varepsilon+\xi\left[f^{-1}\left(C_{0}^{i}\right)\right], \quad \mu$-a.s.

By (II.4.1) and (II.4.2), there exists a $\mu$-null set $N_{2} \in \mathscr{O}_{f}$ such that (II.4.3) $\quad \lim _{t \rightarrow \infty} R^{(t)}\left(x, C_{0}^{i}\right)=\xi\left[f^{-1}\left(O_{0}^{i}\right)\right], \quad \forall x \notin N_{1} \cup N_{2}$ and $\nabla C_{0}^{i} \in \mathscr{T}_{0}$ and this limit is uniform with respect to $\left(x, C_{0}^{i}\right)$ on $\mathbb{C}\left(N_{1} \cup N_{2}\right) \times \mathscr{T}_{0}$. $2^{\circ}$ Let us prove now that (II.4.3) is verified for every $C \epsilon \mathscr{T}$. For this purpose, let us prove that the class $\mathscr{T}^{\prime}$ of sets $C_{\epsilon} \mathscr{T}$ such that (II.4.3) is verified, is a monotone class containing $\mathscr{T}_{0}$ (and, consequently, $\mathscr{T}^{\prime}$ contains $\mathscr{T}$ ). Indeed, it is clear that $\mathscr{T}^{\prime}$ contains $\mathscr{T}_{0}$. It remains to prove that $\mathscr{T}^{\prime}$ is a monotone class. Let $\left(C_{i}\right)_{i \in \mathbf{N}^{*}}$ be a monotone sequence of elements of $\mathscr{T}$ whose limit is $O$. For every $x \notin N_{1} \cup N_{2}$, we have

$$
\lim _{t \rightarrow \infty} R^{(t)}(x, C)=\lim _{t \rightarrow \infty} \lim _{i \rightarrow \infty} R^{(t)}\left(x, C_{i}\right),
$$

for $R^{(t)}(x, \cdot)$ is a probability on $\mathscr{T}$. 
Since the $\operatorname{limit} \lim _{t \rightarrow \infty} R^{(t)}\left(x, C_{i}\right)$ is uniform with respect to $C_{i}$, we have (II.4.5)

$$
\begin{aligned}
\lim _{t \rightarrow \infty} R^{(t)}(x, C) & =\lim _{i \rightarrow \infty} \lim _{t \rightarrow \infty} R^{(t)}\left(x, C_{i}\right)=\lim _{i \rightarrow \infty} \xi\left[f^{-1}\left(C_{i}\right)\right] \\
& =\xi\left[f^{-1}\left(\lim _{i \rightarrow \infty} C_{i}\right)\right]=\xi\left[f^{-1}(C)\right], \quad \forall x \notin N_{1} \cup N_{2} .
\end{aligned}
$$

This limit is also uniform with respect to $(x, C)$ on $\mathfrak{\complement}\left(N_{1} \cup N_{2}\right) \times \mathscr{T}$, for, by (II.4.2), $t>n_{\varepsilon}$ and $x \notin N_{1} \cup N_{2}$ implies that

and

$$
-\varepsilon<R^{(t)}\left(x, C_{i}\right)-\xi\left[f^{-1}\left(O_{i}\right)\right]<\varepsilon, \quad \forall i \epsilon \mathbf{N}^{*},
$$

$$
-\varepsilon+\xi\left[f^{-1}\left(C^{(}\right)\right]<R^{(t)}(x, C)<\varepsilon+\xi\left[f^{-1}(C)\right] .
$$

Thus, $C \in \mathscr{T}^{\prime}$, hence $\mathscr{T}^{\prime} \supset \mathscr{T}$, consequently $\mathscr{T}^{\prime}=\mathscr{T}$.

$3^{\circ}$ We examine now separately the three following cases:

(a) $s \geqslant 2$ and $y \notin f\left(N_{1} \cup N_{2}\right)$. Then $f^{-1}(y) \subset \boldsymbol{G}\left(N_{1} \cup N_{2}\right)$ and, by (II.4.5), we have

(II.4.6) $\lim _{t \rightarrow \infty} Q_{s, t}(y, C)=\lim _{t \rightarrow \infty} R^{(t-s)}(x, C)=\xi\left[f^{-1}(C)\right], \quad x \in f^{-1}(y)$, uniformly with respect to $(y, C)$ on $\mathrm{C} f\left(N_{1} \cup N_{2}\right) \times \mathscr{T}$.

(b) $s \geqslant 2$ and $y \in f\left(N_{1} \cup N_{2}\right)$. As indicated in (II.3.1), we take $Q_{s, s+1}(y, C)=\mu^{\prime}(C)$ and, for $t>s+1$, we have

$$
\begin{aligned}
Q_{s, t}(y, C) & =\int_{\mathscr{Q}} Q_{s, s+1}\left(y, d y^{\prime}\right) Q_{s+1, t}\left(y^{\prime}, O\right) \\
& =\int_{\sigma f\left(N_{\mathcal{1}} \cup N_{2}\right)} Q_{s, s+1}\left(y, d y^{\prime}\right) Q_{s_{+1, t}}\left(y^{\prime}, O\right) .
\end{aligned}
$$

By II.4.6 and the Fatou-Lebesgue theorem, we have

(II.4.7) $\quad \lim _{t \rightarrow \infty} Q_{s, t}(y, C)=\int_{\mathrm{bf}\left(N_{\mathbf{1}} \cup N_{2}\right)} d \mu^{\prime}\left(y^{\prime}\right) \lim _{t \rightarrow \infty} Q_{s+1, t}\left(y^{\prime}, C\right)=\xi\left[f^{-1}(C)\right]$,

uniformly with respect to $(y, C)$ on $f\left(N_{1} \cup N_{2}\right) \times \mathscr{T}$.

(c) $s=1$. We have

$$
\lim _{t \rightarrow \infty} Q_{1, t}(y, C)=\int_{\mathscr{Q}} Q_{1,2}\left(y, d y^{\prime}\right) \cdot \lim _{t \rightarrow \infty} Q_{2, t}\left(y^{\prime}, C\right)=\xi\left[f^{-1}(C)\right],
$$

by (II.4.6) and (II.4.7), uniformly with respect to $(y, C)$ on $\mathscr{Y} \times \mathscr{T}$.'

Thus, the Markov process $\left((\mathscr{Y}, \mathscr{T}), Q_{t, t+1}\right)_{t \in \boldsymbol{N}^{*}}$ is strongly and uniformly ergodic.

II.5. HYPOTHESES. Let $\mathscr{Y}^{*}=\left\{y \in \mathscr{Y}: \mu\left[f^{-1}(y)\right]>0\right\}$. We remark that $\mathscr{Y}^{*}$ contains an at most countable set of points, $\mathscr{Y}^{*}=\left\{y_{1}, \ldots, y_{n}, \ldots\right\}$, for $\mu(\mathscr{X})=1$. I.3 (ii) implies that $\mathscr{Y}^{*} \epsilon \mathscr{T}$. We suppose that $\mu\left[f^{-1}\left(\mathscr{Y}^{*}\right)\right]=1$ and that $\nabla x \in \mathscr{X}$, the series $\sum_{k} P_{t}\left(x, f^{-1}\left(y_{k}\right)\right)$ converges uniformly with respect to $t$.

In the particular case where $\mathscr{Y}$ is a finite or countable set, the condition $\mu\left[f^{-1}\left(\mathscr{Y}^{*}\right)\right]=1$ is always verified.

II.6. Proposition. If $\left(X_{t}\right)_{t \in \mathrm{N}^{*}}$ is attached to a strongly ergodic Markov process $\left((\mathscr{X}, \mathscr{B}), P_{t}\right)_{t_{\in} \mathrm{N}^{*}}$ and if, moreover, the hypotheses II.2 and II.5 are satisfied, then $\left(f \circ X_{t}\right)_{t \in \mathbf{N}^{*}}$ is attached to a strongly ergodic Markov process.

Proof. By hypothesis, there exists no $\mu$-null set $N \epsilon \mathscr{B}_{f}$ contained in $f^{-1}\left(\mathscr{Y}^{*}\right) . \forall y_{k} \epsilon \mathscr{Y}^{*}$, there exists a $\mu$-null set $N_{k} \in \mathscr{B}_{f}$ such that

$\lim _{t \rightarrow \infty} R^{(t)}\left(x,\left\{y_{k}\right\}\right)=E_{\bar{\mu}}\left[\lim _{t \rightarrow \infty} P_{t}\left(x, f^{-1}\left(y_{k}\right)\right) \mid \mathscr{B}_{f}\right]=\xi\left[f^{-1}\left(y_{k}\right)\right], \quad \nabla x \notin N_{k}$.

Hence, there exists $N=\bigcup_{k=1}^{\infty} N_{k} \epsilon \mathscr{B}_{f}, \mu$-null, such that

$$
\lim _{t \rightarrow \infty} R^{(t)}\left(x,\left\{y_{k}\right\}\right)=\xi\left[f^{-1}\left(y_{k}\right)\right], \quad \nabla x \notin N .
$$

We must now prove that, $\nabla C \subset \mathscr{Y}^{*}$,

$$
\lim _{t \rightarrow \infty} R^{(t)}(x, C)=\xi\left[f^{-1}(C)\right], \quad \forall x \notin N .
$$

This formula is true for every $C$ which is a finite union of $\left\{y_{k}\right\}$. It remains for us to examine the case where $C$ is a countable union of $\left\{y_{k}\right\}$. Let $C=\bigcup_{j=1}^{\infty}\left\{y_{k_{j}}\right\}$ be such a countable union. We have, $\nabla x \notin N$,

$$
\begin{aligned}
\lim _{t \rightarrow \infty} R^{(t)}(x, C) & =\lim _{t \rightarrow \infty} \sum_{j=1}^{\infty} R^{(t)}\left(x,\left\{y_{k_{j}}\right\}\right) \\
& =\lim _{t \rightarrow \infty} \sum_{j=1}^{\infty} P_{t}\left(x, f^{-1}\left(y_{k_{j}}\right)\right) \\
& =\sum_{j=1}^{\infty} \lim _{t \rightarrow \infty} P_{t}\left(x, f^{-1}\left(y_{k_{j}}\right)\right) \\
& =\sum_{j=1}^{\infty} \lim _{t \rightarrow \infty} R^{(t)}\left(x,\left\{y_{k_{j}}\right\}\right) \\
& =\sum_{j=1}^{\infty} \xi\left[f^{-1}\left(y_{k_{j}}\right)\right] \\
& =\xi\left[f^{-1}(C)\right] .
\end{aligned}
$$

For the rest of the proof, we use the same argument as that in II. $4.3^{\circ}$ with the three cases: $s \geqslant 2$ and $y \in \mathfrak{Q} f(N) ; s \geqslant 2$ and $y \in f(N) ; s \geqslant 1$. 
II.7. Proposition. If $\left(X_{t}\right)_{t \in N^{*}}$ is attached to a weakly ergodic homogeneous Markov process, and if II.2 and II.5 are satisfied, then $\left(f \circ X_{t}\right)_{t \in \mathrm{N}^{*}}$ is attached to a weakly ergodic Markov process $\left((\mathscr{Y}, \mathscr{T}), Q_{t, t+1}\right)_{t \in \mathbf{N}^{*}}$.

Proof. We use, for the proof of this proposition, the following criteria for weak ergodicity (cf. [1]):

A Markov process $\left((\Lambda, \mathscr{L}), q_{t, t+1}\right)_{t \in \mathbf{N}^{*}}$ is weakly ergodic if and only if for every $s \in \boldsymbol{N}^{*}, B \epsilon \mathscr{L}$, for every increasing sequence $\left(t_{j}\right)_{j_{\epsilon \mathbf{N}^{*}}}$ of indices such that $q_{s, t_{j}}(u, B)$ converges to a limit when $j \rightarrow \infty$, for some $u \in \Lambda, q_{s, t_{j}}(v, B)$ converges $\nabla v \in \Lambda$ to the same limit; moreover, this common limit is independent of $s$.

$1^{0}$ Let $\left(x_{0}, C\right) \in \mathscr{X} \times \mathscr{T}$, and let $\sigma=\left(t_{i}\right)_{i \in N^{*}}$ be an increasing sequence of indices such that the sequence $\left(P_{t_{i}}\left(x_{0}, f^{-1}(C)\right)\right)_{i \in \mathbf{N}^{*}}$ is convergent. The criteria cited above implies that $\forall s \in N^{*}$ and $\forall x \in \mathscr{X}$, the sequence $\left(P_{t_{i}-s}\left(x, f^{-1}(C)\right)\right)_{i_{\mathbf{N}^{*}}}$ converges to the same limit. Let $l(\sigma, C)$ denote the common limit of these sequences.

An argument analogous to that in II.6.1 $1^{\circ}$ shows that

$$
\lim _{i \rightarrow \infty} R^{\left(t_{i}-s\right)}(\cdot, C)=l(\sigma, C), \quad \mu \text {-a.s. }
$$

and since there exists no $\mu$-null element of $\mathscr{B}_{f}$, contained in $f^{-1}\left(\mathscr{Y}^{*}\right)$, we have

$$
\lim _{i \rightarrow \infty} R^{\left(t_{i}-8\right)}(x, C)=l(\sigma, C), \quad \nabla x \in f^{-1}\left(\mathscr{Y}^{*}\right) .
$$

Using the same argument employed in II.4.3 $3^{\circ}$, with the 3 cases (a), (b), (c), we have $\nabla C \epsilon \mathscr{T}, \forall \sigma$,

$$
\lim _{i \rightarrow \infty} Q_{8, t_{i}}(y, O)=l(\sigma, O), \quad \forall s \in \boldsymbol{N}^{*} \text { and } \forall y \in \mathscr{Y} .
$$

$2^{\circ}$ Now let $s_{0} \in \boldsymbol{N}^{*}, O \epsilon \mathscr{T}$ and let $\sigma=\left(t_{j}\right)_{j_{\epsilon \mathbf{N}^{*}}}$ be a sequence of indices such that $\left(Q_{s_{0}, t_{j}}\left(y_{0}, O\right)\right)_{j_{\epsilon} \mathbf{N}^{*}}$ is convergent for a certain $y_{0} \in \mathscr{Y}$. Let $l\left(\sigma, s_{0}, y_{0}, C\right)$ denote this limit. Following the criteria, we must prove that this limit is independent of $y_{0}$ and $s_{0}$. Let us examine separately the 3 following eases:

(a) $s \geqslant 2$ and $y_{0} \epsilon \mathscr{Y}^{*}$. Then, we have

and

$$
Q_{s_{0}, t_{j}}\left(y_{0}, C\right)=R^{\left(t_{j}-s_{0}\right)}\left(x_{0}, C\right), \quad x_{0} \in f^{-1}\left(y_{0}\right),
$$

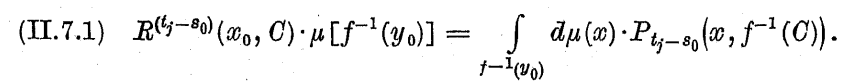

The sequence $\left(P_{t_{j}-s_{0}}\left(x_{0}, f^{-1}(C)\right)\right)_{j \in \mathbf{N}^{*}}$ being a sequence of numbers contained in the interval $[0,1]$, we can find a convergent subsequence $\left(P_{t_{t_{k}}-s_{0}}\left(x_{0} ; f^{-1}(C)\right)\right)_{k \in \mathbf{N}^{*}}$. Since $\left((\mathscr{X}, \mathscr{B}), P_{t}\right)_{t \in \mathbf{N}^{*}}$ is weakly ergodic, the limit of this convergent subsequence is independent of $x_{0}$. and of $s_{0}$. Let $l\left(\sigma^{\prime}, C\right)$ denote this limit, $\sigma^{\prime}$ being the subsequence $\left(t_{j_{k}}\right)_{k_{\in} \mathbf{N}^{*}}$.

By the Fatou-Lebesgue theorem, (II.7.1) implies that

$$
\lim _{j \rightarrow \infty} R^{\left(t_{j_{k}}-s_{0}\right)}\left(x_{0}, C\right) \cdot \mu\left[f^{-1}\left(y_{0}\right)\right]=\int_{f^{-1}\left(y_{0}\right)} d \bar{\mu}(x) \cdot \lim _{k \rightarrow \infty} P_{t_{j_{k}}-s_{0}}\left(x, f^{-1}(C)\right),
$$

hence

$$
l\left(\sigma, s_{0}, y_{0}, C\right) \cdot \mu_{0}\left[f^{-1}\left(y_{0}\right)\right]=l\left(\sigma^{\prime}, C\right) \cdot \mu\left[f^{-1}\left(y_{0}\right)\right] .
$$

Consequently, since $\mu\left[f^{-1}\left(y_{0}\right)\right]>0$,

$$
l\left(\sigma, s_{0}, y_{0}, C\right)=l\left(\sigma^{\prime}, C\right) .
$$

Thus, all the convergent subsequences of the sequence $\left(P_{t_{j}-s_{0}}\left(x_{0}, f^{-1}(C)\right)\right)_{i \in \mathbb{N}^{*}}$ have the same limit: the sequence itself is then convergent, and

$$
\lim _{j \rightarrow \infty} P_{t_{j}-s_{0}}\left(x_{0}, f^{-1}(C)\right)=l\left(\sigma, s_{0}, y_{0}, C\right) .
$$

By $1^{\circ} \nabla s \in \boldsymbol{N}^{*}$ and $\nabla y \in \mathscr{Y}$ :

$$
\lim _{j \rightarrow \infty} Q_{s, t_{j}}(y, C)=\lim _{j \rightarrow \infty} Q_{s_{0}, t_{j}}\left(y_{0}, C\right) .
$$

(b) $s_{0} \geqslant 2$ and $y_{0} \oiint_{\mathscr{Y}^{*}}$. Then, $f^{-1}\left(y_{0}\right) \subset \boldsymbol{G} f^{-1}\left(\mathscr{Y}^{*}\right)$. We have

$$
Q_{s_{0}, t_{j}}\left(y_{0}, C\right)=\int_{\mathscr{Q} *} d \mu^{\prime}(y) Q_{s_{0}+1, t_{j}}(y, C)
$$

Let $y_{1} \in \mathscr{Y}^{*}$, and let $\left(Q_{s_{0}+1, t_{j_{k}}}\left(y_{1}, C\right)\right)_{k \in \mathbb{N}^{*}}$ be a convergent subsequence. By (a), $\nabla y \in \mathscr{Y}$, the sequence $\left(\left(Q_{s_{0}+1, t_{j_{k}}}(y, C)\right)\right)_{k_{\epsilon} \mathbf{N}^{*}}$ converges to the same limit, the Fatou-Lebesgue theorem gives

$$
\lim _{k \rightarrow \infty} Q_{s_{0}, t_{j_{k}}}\left(y_{0}, C\right)=\int_{\mathbb{g}^{*}} d \mu^{\prime}(y) \cdot \lim _{k \rightarrow \infty} Q_{s_{0}+1, t_{j_{k}}}(y, C),
$$

that is to say

$$
l\left(\sigma, s_{0}, y_{0}, C\right)=\lim _{k \rightarrow \infty} Q_{s_{0}+1, t_{j_{k}}}\left(y_{1}, C\right) .
$$

Thus, all the convergent subsequences of the sequence $\left(Q_{s_{0}+1, t_{j}}\left(y_{0}, C\right)\right)_{j \in \mathbf{N}^{*}}$ have the same limit. Hence, the sequence itself is convergent, and

$$
\lim _{j \rightarrow \infty} Q_{s_{0}+1, t_{j}}\left(y_{1}, C\right)=l\left(\sigma, s_{0}, y_{0}, C\right) .
$$

By $1^{\circ} \nabla s \epsilon N^{*}$ and $\nabla y \epsilon \mathscr{Y}$,

$$
\lim _{j \rightarrow \infty} Q_{s, t_{j}}(y, C)=\lim _{j \rightarrow \infty} Q_{s, t_{j}}\left(y_{0}, C\right) \text {. }
$$

(c) $s_{0}=1$. We have

$$
Q_{1, t_{j}}\left(y_{0}, C\right)=\int_{\mathscr{Q}} Q_{1,2}\left(y_{0}, d y\right) Q_{2, t_{j}}(y, C)
$$


Let $y_{2} \epsilon \mathscr{Y}$ and let $\left(Q_{2, t_{j_{k}}}\left(y_{2}, C\right)\right)_{k \in \mathbf{N}^{*}}$ be a convergent subsequence. By (a) and (b), the sequence $\left(Q_{2, t_{j_{k}}}(y, C)\right)_{k \in \boldsymbol{N}^{*}}$ converges to a limit independent of $y \epsilon \mathscr{Y}$. The Fatou-Lebesgue theorem shows that

$$
\lim _{k \rightarrow \infty} Q_{1, t_{j_{k}}}\left(y_{0}, C\right)=\int_{\mathscr{V}} Q_{1,2}\left(y_{0}, d y\right) \lim _{k \rightarrow \infty} Q_{2, t_{j_{k}}}(y, C),
$$

viz.

$$
l\left(\sigma, s_{0}, y_{0}, C\right)=\lim _{k \rightarrow \infty} Q_{2, t_{j_{k}}}\left(y_{2}, C\right) .
$$

All the convergent subsequences of $\left(Q_{2, t_{j}}\left(y_{2}, C\right)\right)_{j_{\epsilon} \boldsymbol{N}^{*}}$ having the same limit, the sequence itself is convergent, and we have

Consequently,

$$
\lim _{j \rightarrow \infty} Q_{2, t_{j}}\left(y_{2}, C\right)=l\left(\sigma, s_{0}, y_{0}, C\right)
$$

$$
\lim _{j \rightarrow \infty} Q_{1, t_{j}}(y, C)=l\left(\sigma, s_{0}, y_{0}, C\right)=\lim _{j \rightarrow \infty} Q_{1, t_{j}}\left(y_{0}, C\right) .
$$

Thus, in every case, $l\left(\sigma, s_{0}, y_{0}, C\right)$ is independent of $s_{0}$ and $y_{0}$, and the Markov process $\left((\mathscr{Y}, \mathscr{T}), Q_{t, t+1}\right)_{t_{\epsilon} \mathbf{N}^{*}}$ is weakly ergodic.

II.8. ExAMPLES. Here are some examples in the case of a finite state space.

$1^{\circ} \mathscr{X}=\{1,2,3,4\}, \mathscr{B}=\mathscr{P}(\mathscr{X})$ and

$$
P=\left(\begin{array}{cccc}
\frac{1}{3} & \frac{1}{3} & \frac{1}{6} & \frac{1}{6} \\
\frac{1}{4} & \frac{1}{4} & \frac{1}{4} & \frac{1}{4} \\
\frac{1}{6} & \frac{1}{6} & \frac{1}{3} & \frac{1}{3} \\
\frac{2}{10} & \frac{2}{10} & \frac{3}{10} & \frac{3}{10}
\end{array}\right) .
$$

We can verify that the process $\left((\mathscr{X}, \mathscr{P}(\mathscr{X})), P_{t}\right)_{t \in N^{*}}$ is strongly and uniformly ergodic, for $\mathscr{X}$ is finito and

$$
\lim _{t \rightarrow \infty} P_{t}=\left(\begin{array}{llll}
\frac{11}{47} & \frac{11}{47} & \frac{25}{94} & \frac{25}{94} \\
\frac{11}{47} & \frac{11}{47} & \frac{25}{94} & \frac{25}{94} \\
\frac{11}{47} & \frac{11}{47} & \frac{25}{94} & \frac{25}{94} \\
\frac{11}{47} & \frac{11}{47} & \frac{25}{94} & \frac{25}{94}
\end{array}\right) .
$$

Now, consider $f: \mathscr{X} \rightarrow \mathscr{Y}$ such that $f(\mathcal{1})=f(2)=1 ; f(3)=f(4)=2$. We take $\mu$ on $\mathscr{B}_{f}$ such that

$$
\mu\left[f^{-1}(i)\right]=\sum_{j \in \mathscr{X}} \alpha_{j} P\left(j, f^{-1}(i)\right), \quad \nabla i \epsilon \mathscr{Y} \text { where } \alpha_{j}>0, \sum_{j \in \mathscr{X}} a_{j}=1 .
$$

Hypothesis II.2, in our case, is satisfied by the mapping $R: \mathscr{X} \times \mathscr{P}(\mathscr{Y}) \rightarrow$ $\rightarrow[0,1]$ defined by

$$
\begin{array}{lll}
R\left(j_{0},\{1\}\right)=\frac{7}{12}, & R\left(j_{0},\{2\}\right)=\frac{5}{12} & \text { for } j_{0}=1,2, \\
R\left(j_{0},\{1\}\right)=\frac{11}{30}, & R\left(j_{0},\{2\}\right)=\frac{19}{30} & \text { for } j_{0}=3,4 .
\end{array}
$$

Then, we have

$$
Q_{t, t+1}=\left(\begin{array}{cc}
\frac{7}{12} & \frac{5}{12} \\
\frac{11}{30} & \frac{19}{30}
\end{array}\right), \quad \nabla t \geqslant 2 .
$$

Computations show that

$$
\lim _{t \rightarrow \infty} Q_{s, t}=\left(\begin{array}{cc}
\frac{22}{47} & \frac{25}{47} \\
\frac{11}{30} & \frac{19}{30}
\end{array}\right), \quad \forall s \geqslant 2,
$$

and

$$
\lim _{t \rightarrow \infty} Q_{1, t}=Q_{1,2} \cdot \lim _{t \rightarrow \infty} Q_{2, t}=\lim _{t \rightarrow \infty} Q_{2, t},
$$

for $Q_{1,2}$ is a stochastic matrix and $\lim Q_{2, t}$ is with all its rows identical. Thus, $\left((\mathscr{Y}, \mathscr{P}(\mathscr{Y})), Q_{t, t+1}\right)_{t_{\in} \boldsymbol{N}^{*}}$ is also strongly and uniformly ergodic. $2^{\circ} \mathscr{X}=\{1,2,3\}, \mathscr{B}=\mathscr{P}(\mathscr{X})$ and

$$
P=\left(\begin{array}{ccc}
\frac{1}{2} & \frac{1}{2} & 0 \\
\frac{1}{2} & \frac{1}{2} & 0 \\
\frac{1}{2} & \frac{1}{2} & 0
\end{array}\right) .
$$

The Markov process $\left((\mathscr{X}, \mathscr{P}(\mathscr{X})), P_{t}\right)_{t \in \mathbf{N}^{*}}$ is strongly and uniformly ergodic, for $\mathscr{X}$ is finite and $P_{t}=P, \nabla t \in N^{*}$. Let $\mathscr{Y}=\{1,2\}$ and $f: \mathscr{X} \rightarrow \mathscr{Y}$ bo defined by $f(1)=f(2)=1$, and $f(3)=2$.

We take $\mu$ on $\mathscr{B}_{f}$ such that

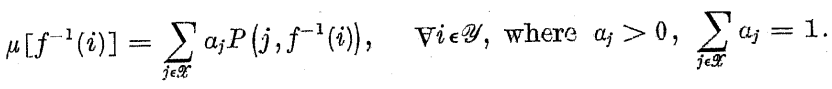

Thus,

$$
\mu\left[f^{-1}(1)\right]=1 \quad \text { and } \quad \mu\left[f^{-1}(2)\right]=0 .
$$

Hypothesis II.2 is satisfied by the mapping $R: \mathscr{X} \times \mathscr{P}(\mathscr{Y}) \rightarrow[0,1]$ defined by

$$
\begin{aligned}
& R(1,\{1\})=R(2,\{1\})=1, \\
& R(1,\{2\})=R(2,\{2\})=0, \\
& R(3,\{1\})+R(3,\{2\})=1,
\end{aligned}
$$

where $R(3,\{1\})$ can be arbitrarily chosen. 
Hence, we can take

$$
Q_{t, t+1}=\left(\begin{array}{cc}
1 & 0 \\
v\left[f^{-1}(1)\right] & v\left[f^{-1}(2)\right]
\end{array}\right) \text { for } t \geqslant 2,
$$

where $\nu$ is an arbitrarily chosen probability measure on $\mathscr{B}_{f}$.

a) If $\nu$ is such that $v\left[f^{-1}(1)\right]=0$ and $\nu\left[f^{-1}(2)\right]=1$, then

$$
Q_{t, t+1}=\left(\begin{array}{ll}
1 & 0 \\
0 & 1
\end{array}\right), \quad \forall t \geqslant 2
$$

and the process $\left((\mathscr{Y}, \mathscr{P}(\mathscr{Y})), Q_{t, t+1}\right)_{t \in \mathbf{N}^{*}}$ is not strongly ergodic.

b) If $\nu$ is such that $\nu\left[f^{-1}(1)\right]=\alpha$, where $\left.\left.\alpha \epsilon\right] 0,1\right]$, then

$$
Q_{t, t+1}=\left(\begin{array}{cc}
1 & 0 \\
\alpha & 1-\alpha
\end{array}\right), \quad \forall t \geqslant 2
$$

We have

$$
\lim _{t \rightarrow \infty} Q_{s, s+t}=\lim _{t \rightarrow \infty}\left(\begin{array}{cc}
1 & 0 \\
1-(1-\alpha)^{t} & (1-\alpha)^{t}
\end{array}\right)=\left(\begin{array}{ll}
1 & 0 \\
1 & 0
\end{array}\right), \quad \nabla s \geqslant 2,
$$

and, consequently, the process $\left((\mathscr{Y}, \mathscr{P}(\mathscr{Y})), Q_{t, t+1}\right)_{t_{\in} N^{*}}$ is strongly ergodic.

We examine now the converse for the proposition II.7.

II.9. HrPotheses. (i) $\nabla x \in \mathscr{X}, P(x, \cdot)$ is of the form

$$
P(x, B)=\int_{B} g\left(x, x^{\prime}\right) d \bar{\mu}\left(x^{\prime}\right),
$$

where $B \in \mathscr{B}$, and $g(x, \cdot)$ is $\mathscr{B}_{f}$-measurable.

(ii) $\nabla(x, B) \epsilon \mathscr{X} \times \mathscr{B}$, if $\left(t_{j}\right)_{j_{\epsilon N^{*}}}$ is an increasing sequence of indices such that the sequence $\left(P_{t_{j}}(x, B)\right)_{j \in N^{*}}$ converges, then $\forall x^{\prime} \in f^{-1}(f(x))$, the sequence $\left(P_{t_{j}}\left(x^{\prime}, B\right)\right)_{i_{\epsilon} \boldsymbol{N}^{*}}$ converges and

$$
\text { (II.9.2) } \quad \lim _{j \rightarrow \infty} P_{t_{j}}(x, B)=\lim _{j \rightarrow \infty} P_{t_{j}}\left(x^{\prime}, B\right) \text {. }
$$

II.10. The following is an interpretation of II.9: II.9 (i) implies the condition of Doeblin (cf. [2]) for the homogeneous Markor process $\left((\mathscr{X}, \mathscr{B}), P_{t}\right)_{t \in \boldsymbol{N}^{*}}$. II 9. (ii) implies that $\nabla y \in \mathscr{Y}$, if $f^{-1}(y)$ is included in an ergodic set (cf. [2]) decomposable in cyclic subsets $\Gamma_{1}, \ldots, \Gamma_{d}$, then $f^{-1}(y)$ is included in one of these cyclic subsets. Indeed, suppose that there exist two distinct cyclic subsets $\Gamma_{k}$ and $\Gamma_{k^{\prime}}$ such that $\Gamma_{k} \cap f^{-1}(y) \neq \varnothing$ and $\Gamma_{k^{\prime}} \cap f^{-1}(y) \neq \varnothing$. We know, by [2], that there exist probabilities ${ }_{k} \pi$ and $k_{k^{\prime}} \pi$ on $\mathscr{B}$ such that ${ }_{k} \pi\left(\Gamma_{k}\right)=1$ and ${ }_{k^{\prime}} \pi\left(\Gamma_{k^{\prime}}\right)=1$ and that

$$
\begin{aligned}
& \lim _{n \rightarrow \infty} P_{n d}\left(x_{k}, \Gamma_{k}\right) \doteq{ }_{k} \pi\left(\Gamma_{k}\right)=1, \\
& \lim _{n \rightarrow \infty} P_{n d}\left(x_{k^{\prime}}, \Gamma_{k^{\prime}}\right)={ }_{k^{\prime}} \pi\left(\Gamma_{k^{\prime}}\right)=0,
\end{aligned}
$$

and thus, II.9 (ii) is not be" verified.
II.11. Propostmion. Under II.9 (i), $\forall t \in \mathbf{N}^{*}$ and $\forall(x, B) \epsilon \mathscr{X} \times \mathscr{B}$,

$$
P_{t}(x, B)=\int_{B} g^{(t)}\left(x, x^{\prime}\right) d \bar{\mu}\left(x^{\prime}\right),
$$

where the $g^{(t)}, \mathscr{B}_{f}$-measurable, are defined by recurrence:

$$
\begin{gathered}
g^{(1)}=g, \\
g^{(t)}\left(x, x^{\prime}\right)=\int_{\mathscr{X}} g^{(t-1)}\left(x, x_{1}\right) g\left(x_{1}, x^{\prime}\right) d \bar{\mu}\left(x_{1}\right), \quad \forall x \text { and } x^{\prime} \in \mathscr{X} .
\end{gathered}
$$

Proof. We proceed by induction. It is easy to verify the formula for $t=2$. Suppose now that

$$
P_{t-1}(x, B)=\int_{B} g^{(t-1)}\left(x, x^{\prime}\right) d \bar{\mu}\left(x^{\prime}\right) .
$$

We have

$$
\begin{aligned}
P_{t}(x, B) & =\int_{\mathscr{X}} P_{t-1}\left(x, d x_{1}\right) P\left(x_{1}, B\right) \\
& =\int_{\mathscr{X}} g^{(t-1)}\left(x, x_{1}\right) d \bar{\mu}\left(x_{1}\right) \cdot \int_{\mathcal{B}} g\left(x_{1}, x^{\prime}\right) d \bar{\mu}\left(x^{\prime}\right) \\
& =\int_{B}\left[\int_{\mathscr{X}} g^{(t-1)}\left(x, x_{1}\right) g\left(x_{1}, x^{\prime}\right) d \bar{\mu}\left(x_{1}\right)\right] d \bar{\mu}\left(x^{\prime}\right) \\
& =\int_{\mathcal{B}} g^{(t)}\left(x, x^{\prime}\right) d \bar{\mu}\left(x^{\prime}\right) .
\end{aligned}
$$

II.12. PRoposirion. Suppose that II.5 and II.9 are satisfied. Let $\left((\mathscr{Y}, \mathscr{T}), Q_{t, t+1}\right)_{t \in \mathbf{N}^{*}}$, to which $\left(f \circ X_{t}\right)_{t_{\epsilon \mathbf{N}}}$ is attached, be defined by (II. 3.1) with $N=f^{-1}\left(\mathscr{Y}^{*}\right)$. If $\left((\mathscr{Y}, \mathscr{T}), Q_{t, t+1}\right)_{t \in \mathbf{N}^{*}}$ is strongly ergodic, then $\left((\mathscr{X}, \mathscr{B}), P_{t}\right)_{\epsilon \in \mathbf{N}^{*}}$ is also strongly ergodic.

Proof. By hypothesis, $\forall s \in \boldsymbol{N}^{*}, \forall y \in \mathscr{Y}$ and $\forall C \epsilon \mathscr{T}$,

(II.12.1) $\lim _{t \rightarrow \infty} Q_{s, s+t}(y, C)=\pi(C), \quad$ where $\pi$ is a probability on $\mathscr{T}$.

$1^{\circ}$ At first, let us examine the limit $\lim _{t \rightarrow \infty} P_{t}(x, B)$, for $x \in \mathscr{X}$ and $B \epsilon \mathscr{B}_{f}$.

Let $x_{0} \in \mathscr{X}, y_{0}=f\left(x_{0}\right)$ and $O \in \mathscr{T}$.

(a) If $x_{0} \in f^{-1}\left(\mathscr{Y}^{*}\right)$, then $f^{-1}\left(y_{0}\right) \subset f^{-1}\left(\mathscr{Y}^{*}\right)$, and

$$
Q_{s+t}\left(y_{0}, C\right)=R^{(t)}\left(x_{0}, C\right) \text {. }
$$

Hence,

$$
\text { (II.12.2) } Q_{s, s+t}\left(y_{0}, C\right) \cdot \mu\left[f^{-1}\left(y_{0}\right)\right]=\int_{t-1} \overline{d\left(y_{0}\right)}(x) P_{t}\left(x, f^{-1}(C)\right) \text {. }
$$

$\left(P_{t}\left(x_{0}, f^{-1}(C)\right)\right)_{t \in \mathbf{N}^{*}}$ being a sequence of numbers of $[0,1]$, we can find a convergent subsequence $\left(P_{t_{j}}\left(x_{0}, f^{-1}(C)\right)\right)_{j \in \mathbf{N}^{*}}$ whose limit is $l\left(x_{0}, \sigma, C\right)$, 
$\sigma$ denoting the increasing sequence $\left(t_{j}\right)_{j_{\epsilon} \mathbf{N}^{*}}$ of indices. By II.9 (ii), we have $\forall x \in f^{-1}\left(y_{0}\right)$,

(II.12.3)

$$
\lim _{j \rightarrow \infty} P_{t_{j}}\left(x, f^{-1}(C)\right)=l\left(x_{0}, \sigma, C\right) .
$$

Consequently, the Fatou-Lebesgue theorem, (II.12.1), (II.12.2) and (II.12.3) imply that

$$
\lim _{j \rightarrow \infty} Q_{s, s+t_{j}}\left(y_{0}, C\right) \cdot \mu\left[f^{-1}\left(y_{0}\right)\right]=\int_{f^{-1}\left(y_{0}\right)} d \bar{\mu}(x) \cdot \lim _{j \rightarrow \infty} P_{t_{j}}\left(x, f^{-1}(O)\right),
$$

viz.

$$
\lim _{j \rightarrow \infty} Q_{s, s+t_{j}}\left(y_{0}, C\right) \cdot \mu\left[f^{-1}\left(y_{0}\right)\right]=l\left(x_{0}, \sigma, C\right) \cdot \mu\left[f^{-1}\left(y_{0}\right)\right]
$$

viz.

$$
\pi(C)=l\left(x_{0}, \sigma, C\right), \quad \text { for } \quad \mu\left[f^{-1}\left(y_{0}\right)\right]>0 .
$$

All the convergent subsequences of the sequence $\left(P_{t}\left(x_{0}, f^{-1}(C)\right)\right)_{t \in \mathbf{N}^{*}}$ having the same limit, the sequence itself is then convergent and we have

(II.12.4) $\quad \lim _{t \rightarrow \infty} P_{t}\left(x_{0}, f^{-1}(C)\right)=\pi(C), \quad \forall x_{0} \epsilon f^{-1}\left(\mathscr{Y}^{*}\right)$ and $\forall C \epsilon \mathscr{T}$.

(b) If $x_{0} \in \mathbb{Q} f^{-1}\left(\mathscr{Y}^{*}\right)$, we have, for $t \geqslant 2$,

$$
P_{t}\left(x_{0}, f^{-1}(C)\right)=\int_{\mathscr{X}} P\left(x_{0}, d x\right) P_{t-1}\left(x, f^{-1}(C)\right)
$$

Since $\mu\left[f^{-1}\left(\mathscr{Y}^{*}\right)\right]=1$, we have $P\left(x, \dot{f}^{-1}\left(\mathscr{Y}^{*}\right)\right)=1, \forall x \in \mathscr{X}$. Hence,

$$
P_{t}\left(x_{0}, f^{-1}(C)\right)=\int_{f^{-1}\left(\mathscr{U}^{*}\right)} P\left(x_{0}, d x\right) P_{t-1}\left(x, f^{-1}(C)\right)
$$

so that, by (II.12.4) and the Fatou-Lebesgue theorem,

(II.12.5) $\quad \lim _{t \rightarrow \infty} P_{t}\left(x_{0}, f^{-1}(C)\right)=\int_{f^{-1}\left(\mathscr{G}^{*}\right)} P\left(x_{0}, d x\right) \lim _{t \rightarrow \infty} P_{t-1}\left(x, f^{-1}(C)\right)=\pi(C)$.

Thus, (a) and (b) show that $\forall(x, C) \in \mathscr{X} \times \mathscr{T}$,

$$
\lim _{t \rightarrow \infty} P_{t}\left(x, f^{-1}(C)\right)=\pi(C) \text {. }
$$

$2^{0}$ We examine now the $\operatorname{limit} \lim P_{t}(x, B)$ for $x \in \mathscr{X}$ and $B \in \mathscr{B}$. Let $x$ and $x^{\prime} \in \mathscr{X}$, and $y^{\prime}=f\left(x^{\prime}\right)$. By (II.12.6) and II.10, we have

$$
\lim _{t \rightarrow \infty} P_{t}\left(x, f^{-1}\left(y^{\prime}\right)\right)=\pi\left(\left\{y^{\prime}\right\}\right)=\lim _{t \rightarrow \infty} \int_{j^{-1}\left(y^{\prime}\right)} g^{(t)}\left(x, x_{1}\right) d \bar{\mu}\left(x_{1}\right) .
$$

$g^{(t)}(x, \cdot)$ being by hypothesis $\mathscr{B}_{f}$-measurable, is then constant on $f^{-1}\left(y^{\prime}\right)$ which is an atom of $\mathscr{B}_{f}$. Hence,

$$
\pi\left(\left\{y^{\prime}\right\}\right)=\lim _{t \rightarrow \infty} g^{(t)}\left(x, x^{\prime}\right) \cdot \mu\left[f^{-1}\left(y^{\prime}\right)\right]
$$

We then conclude that, $\forall x \in \mathscr{X}$, the sequence $\left(g^{(t)}(x, \cdot)\right)_{t \in \mathbf{N}^{*}}$ converges $\mu$-a.s. to a limit $g^{\prime}$ independent of $x \in \mathscr{X}$.

Consequently,

$$
\text { (II.12.7) } \begin{aligned}
\lim _{t \rightarrow \infty} P_{t}(x, B) & =\lim _{t \rightarrow \infty} \int_{B} g^{(t)}\left(x, x_{1}\right) d \bar{\mu}\left(x_{1}\right) \\
& =\int_{B} \lim _{t \rightarrow \infty} g^{(t)}\left(x, x_{1}\right) d \bar{\mu}\left(x_{1}\right)=\int_{B} g^{\prime}\left(x_{1}\right) d \bar{\mu}\left(x_{1}\right) .
\end{aligned}
$$

This last equality proves the strong ergodicity of $\left((\mathscr{X}, \mathscr{B}), P_{t, t+1}\right)_{t \in \mathbf{N}^{*}}$.

In fact, we have a stronger result than Proposition II.12, but its proof makes use of the latter.

II. 13. Proposition. Suppose that II.5 and II.9 are satisfied. Let $\left((\mathscr{Y}, \mathscr{T}), Q_{t, t+1}\right)_{t \in \mathbf{N}^{*}}$, to which $\left(f \circ X_{t}\right)_{t \in \mathbf{N}^{*}}$ is attached, be defined by (II.3.1) with $N=f^{-1}\left(\mathscr{Y}^{*}\right)$. If $\left((\mathscr{Y}, \mathscr{T}), Q_{t, t+1}\right)_{t \in \mathbf{N}^{*}}$ is weakly ergodic, then $\left((\mathscr{X}, \mathscr{B}), P_{t}\right)_{t \in \mathbf{N}^{*}}$ is strongly ergodic.

Proof. $1^{0}$ II.9 (i) implies that the Markor process $\left((\mathscr{X}, \mathscr{B}), P_{t}\right)_{t \in \mathbf{N}^{*}}$ verifies the Doeblin condition. The Markov process $\left((\mathscr{Y}, \mathscr{T}), Q_{t, t+1}\right)_{t \in \mathbf{N}^{*}}$ defined by (II.3.1) with $N=f^{-1}\left(\mathscr{Y}^{*}\right)$ is homogeneous. It also verifies the Doeblin condition, for $s \geqslant 2$.

$2^{\circ}$ We know (cf. [1]) that, for a homogeneous Markov process satisfying the Doeblin condition, weak ergodicity and strong ergodicity are equivalent. Consequently, the Markov process $\left((\mathscr{Y}, \mathscr{T}), Q_{t, t+1}\right)_{\substack{t \geqslant 2 \\ t \in N^{*}}}$ is strongly ergodic. Then, $\nabla s \geqslant 2$,

$$
\nabla(y, C) \epsilon \mathscr{Y} \times \mathscr{T}, \quad \lim _{t \rightarrow \infty} Q_{s, s+t}(y, C)=\pi(C) .
$$

$3^{\circ}$ Let us prove now that $\left((\mathscr{Y}, \mathscr{T}), Q_{t, t+1}\right)_{t \in \mathbf{N}^{*}}$ is strongly ergodic. Indeed, for $s=1$, and $\nabla(y, O) \epsilon \mathscr{Y} \times \mathscr{T}$,

$$
\begin{aligned}
\lim _{t \rightarrow \infty} Q_{1,1+t}(y, C) & =\lim _{t \rightarrow \infty} \int_{\mathscr{Q} /} Q_{1,2}\left(y, d y^{\prime}\right) Q_{2,1+t}\left(y^{\prime}, C\right) \\
& =\int_{\mathscr{V}} Q_{1,2}\left(y, d y^{\prime}\right) \lim _{t \rightarrow \infty} Q_{2,1+t}\left(y^{\prime}, C\right)=\pi(C) .
\end{aligned}
$$

$4^{\circ}$ By III.12, we see that the process $\left((\mathscr{X}, \mathscr{B}), P_{t}\right)_{t \in N^{*}}$ is strongly ergodic. 


\section{References}

[1] Bùi-Trong.Liê̂u et M. Dorel, Sur le comportement asymptotique de processus de Markov non homogènes, Studia Math. 28 (1967), p. 253-274.

[2] J. L. Doob, Stochastic processes, New York 1953.

[3] P. R. Halmos, and L. J. S avage, Application of the Radon-Nilcodym theorem to the theory of the sufficient statistics, Ann. Math. Statist. 20 (1949), p. 225-241.

[4] J. Neveu, Bases mathématiques du calcul des probabilités, Paris 1964.

[5] M. Rosenblatt, Functions of a Markov process that are Markovian, Journ. Math. Mech. 8 (1959), p. 585-595.

Regu par la Rédaction le 29. 1. 1969

\section{On the zeroes of some random functions}

R. KAUFMAN (Urbana, IIl.)

Let $F(t)$ be a Fourier series with random coefficients and phases,

$$
F(t)=\sum_{n=1}^{\infty} a_{n} X_{n} \cos \left(n t+\Phi_{n}\right) .
$$

Here $\left(X_{n}\right)_{1}^{\infty}$ is a sequence of mutually independent Gaussian variables of type $N(0,1) ;\left(\left.\Phi(n)\right|_{1} ^{\infty}\right.$ is a sequence of mutually independent variables, uniformly distributed upon $[0,2 \pi]$; and the $X$ 's and $\Phi$ 's are mutually independent. (The basic probability space will be denoted $(\Omega, P$ ): ) About the numbers $a_{n}$ we suppose

$$
a_{n}>0, \quad \log a_{n}=-\beta \log n+o(\log n), \quad \text { with } \frac{1}{2}<\beta \leqslant 1 .
$$

Our goal is an estimation of the zero-set of $F, Z(\omega)=\{t: F(t, \omega)=0\}$. THEOREM. Let $B$ be a closed set in $[0,2 \pi]$ of Hausdorff dimension $d$. Then

$$
\begin{gathered}
P\left\{\operatorname{dim}(Z \cap B) \leqslant d-\beta+\frac{1}{2}\right\}=1, \quad a \geqslant \beta-\frac{1}{2}, \\
P\{Z \cap B=\varnothing\}=1, \quad d<\beta-\frac{1}{2}, \\
P\left\{\operatorname{dim}(Z \cap B) \geqslant d_{1}\right\}>0, \quad 0<d_{1}<d-\beta+\frac{1}{2} .
\end{gathered}
$$

In $\$ 1$ we prove a general principle for the lower bound, whose application is dependent upon specific estimates; derived later about $F$. In $\$ 2$ we review some conclusions from [2] about the uniform convergence of $F$ and its modulus of continuity, and we also obtain a technical lemma about the local character of the trajectories of $F$. In $\S 3$ we obtain an upper bound for the dimension, and in $\S 4$ a lower bound is obtained by combining the work of $\S \S 1$ and 2 .

\$1. Let $B$ be a compact set of real numbers and $\mu$ a probability measure in $B$ such that: $[a, a+h]$.

(i) $\mu([a, a+h]) \leqslant C_{1} h^{d}$ for constants $C_{1}, d>0$ and all intervals 